\title{
Evaluating perioperative glycemic status after different types of pancreatic surgeries via continuous glucose monitoring system: a pilot study
}

\author{
Yishen Mao ${ }^{1,2 \#}$, Xingfei Zhao ${ }^{3 \#}$, Lihui Zhou ${ }^{3}$, Bin $\mathrm{Lu}^{4}$, Chen Jin ${ }^{1}$, Deliang Fu ${ }^{1}$, Lie Yao $^{2}, \mathrm{Ji} \mathrm{Li}^{1}$ \\ ${ }^{1}$ Department of Pancreatic Surgery, Huashan Hospital, Shanghai Medical College, Fudan University, Shanghai, China; ${ }^{2}$ Pancreas Disease Institute, \\ Huashan Hospital, Shanghai Medical College, Fudan University, Shanghai, China; ${ }^{3}$ Department of Nursing, Huashan Hospital, Shanghai Medical \\ College, Fudan University, Shanghai, China; ${ }^{4}$ Department of Endocrinology and Metabolism, Huashan Hospital, Shanghai Medical College, Fudan \\ University, Shanghai, China \\ Contributions: (I) Conception and design: Y Mao, X Zhao, L Yao, J Li; (II) Administrative support: D Fu, C Jin, B Lu, J Li; (III) Provision of study \\ materials or patients: Y Mao, X Zhao, D Fu, C Jin, L Yao, J Li; (IV) Collection and assembly of data: Y Mao, X Zhao, L Zhou, L Yao; (V) Data \\ analysis and interpretation: Y Mao, X Zhao, B Lu; (VI) Manuscript writing: All authors; (VII) Final approval of manuscript: All authors. \\ "These authors contributed equally to this work. \\ Correspondence to: Lie Yao. Pancreas Disease Institute, Huashan Hospital, Shanghai Medical College, Fudan University, Shanghai 200040, China. \\ Email: yaolie_sh@163.com; Ji Li. Department of Pancreatic Surgery, Huashan Hospital, Shanghai Medical College, Fudan University, Shanghai \\ 200040, China. Email: liji@huashan.org.cn.
}

\begin{abstract}
Background: Perioperative glycemic status after pancreatic surgery has never been described. However, it's essential for optimal perioperative glucose management and understanding the pathogenesis of new-onset diabetes mellitus (NODM) after pancreatectomy. Continuous glucose monitoring (CGM) system provides us a helpful tool for closely monitoring and studying perioperative glucose change. This study tried to describe and compare perioperative glucose level and glycemic variability between different types of pancreatic surgeries via CGM device.
\end{abstract}

Methods: This study was designed as a prospective observational study. Eighteen patients were enrolled and were grouped by different types of surgery received: control group (CTRL), pancreaticoduodenectomy (PD), distal pancreatectomy (DP), and total pancreatectomy (TP). CGM devices were implanted and initiated right after the surgery. Mean glucose value (MGV), coefficient of variation (CV), mean of daily difference (MODD), continuous overall net glycemic action (CONGA), and time above range (TAR)/time below range (TBR) was compared between groups to assess glucose level and glycemic variability.

Results: TP showed the highest MGV and CV among all groups $(\mathrm{P}<0.001)$, while CTRL showed the lowest $(\mathrm{P}<0.001)$. PD and DP had similar MGV and CV lower than TP but higher than CTRL $(\mathrm{P}<0.001)$. TP had the highest MODD and CONGA, CTRL had the lowest, but no significant differences were found between groups. TP had the highest TAR (24.29\%) and the lowest TBR (1.28\%), while the control group showed the opposite. The differences in TAR/TBR between groups were all significant $(\mathrm{P}<0.05)$.

Conclusions: TP had the highest mean glucose level and the greatest glycemic variability. PD and DP had similar results: a higher mean glucose level than control but lower than TP. For glycemic variability, PD and DP seemed to have a near-normal result resembling the control group. CGM is useful for glucose monitoring in the perioperative management of pancreatic surgery.

Keywords: Continuous glucose monitoring (CGM); pancreatic surgery; diabetes mellitus; glycemic variability

\footnotetext{
^ ORCID: 0000-0002-0985-0306.
} 
Submitted Jul 26, 2021. Accepted for publication Sep 15, 2021.

doi: $10.21037 / g s-21-495$

View this article at: https://dx.doi.org/10.21037/gs-21-495

\section{Introduction}

Optimal perioperative glucose control is crucial for surgical patients during the perioperative period, as both hyperglycemia or hypoglycemia might lead to a higher incidence of surgical site infection (SSI) and poor surgical outcomes (1-3). For pancreatic surgery, perioperative intensive insulin therapy might also help to lower the incidence of postoperative pancreatic fistula (POPF) (4).

However, it's usually challenging to maintain the glucose level in the optimal range for patients after pancreatic surgeries. Pancreatic surgery and underlying pancreas disease might hamper a patient's glucose metabolism by directly reducing insulin secretion. In the meanwhile, most patients remain normal insulin sensitivity $(5,6)$. This makes patients more vulnerable to glycemic change and results in a higher risk for both hypoglycemic and hyperglycemic events. Besides, different types of pancreatic surgeries might have different impacts on a patient's glucose metabolism $(7,8)$, thus making perioperative glycemic variability more unpredictable.

Therefore, it's relatively essential to know the perioperative glycemic status for different types of pancreatic surgeries. It might reveal different patterns of glucose fluctuation between different surgical types and help identify more extreme glucose values during the perioperative period, which was helpful for surgeons to make an optimal plan for perioperative glucose management (9). In addition to that, perioperative glycemic variability might also be beneficial for exploring the pathogenesis of new-onset diabetes mellitus (NODM) after pancreatectomy and recognizing potential early triggers for this disease $(10,11)$.

Continuous glucose monitoring system (CGM) utilizes a subcutaneous sensor to monitor the glucose level in the interstitial fluid (12). It can continuously monitor realtime interstitial glucose and record the glucose value every 15 minutes for a maximum of 14 days. CGM has been widely used to improve glycemic control in outpatients with diabetes mellitus (12-14). It also has been introduced for inpatients in the intensive care unit (ICU), or those receiving cardiac or neurosurgeries (15-18). The characteristics of CGM above making it especially suitable for monitoring and evaluating the perioperative glycemic status after pancreatic surgery: all glucose values recorded every 15 minutes can help us to know the pattern of glycemic fluctuation after surgery, and the real-time monitoring is able to recognize potential hyper- or hypoglycemic attacks and avoid serious complications.

Nevertheless, only a few studies are applying CGM to patients after pancreatic surgeries. Recent two studies used CGM to investigate the long-term postoperative, rather than perioperative, glycemic status for pancreaticoduodenectomy (PD), distal pancreatectomy (DP), and total pancreatectomy (TP) $(7,8)$. To the extent of our knowledge, the perioperative glycemic status has never been described for pancreatic surgeries so far.

In this pilot study, we tried to describe and compare the different patterns of perioperative glycemic status for three different types of pancreatectomy (PD, DP, and TP) via CGM device, hoping to provide some information for further study the complex glucose metabolism after pancreatectomy. We present the following article in accordance with the STROBE reporting checklist (available at https://dx.doi.org/10.21037/gs-21-495).

\section{Methods}

\section{Etbics statement}

The study was conducted at the department of pancreatic surgery, Huashan Hospital, Fudan University, Shanghai, China, in accordance with the Declaration of Helsinki (as revised in 2013). The study was approved by the Clinical Ethics Committee of Huashan Hospital and registered at chictr.org.cn (ChiCTR2000030177). All patients included agreed to donate their data for research voluntarily and provided written informed consent on admission.

\section{Patients and study design}

The study was designed as a prospective, observational study. Patients were prospectively recruited from in-patients who planned to receive surgical therapy at our department from May to September in 2020. All patients included should fulfill the following criteria: (I) aged from 18 to 75; (II) no history of diabetes mellitus; (III) fasting plasma 
glucose $<7.0 \mathrm{mmol} / \mathrm{L}$ and $\mathrm{HbA} 1 \mathrm{c}<6.5 \%$ on admission; (IV) planned to receive an inerratic pancreatectomy (including PD, DP, and TP) or a palliative surgery without pancreatectomy (like gastrojejunostomy or "open-close" laparotomy). Patients with the following characteristics were excluded: (I) previous history of any types of pancreatic surgery; (II) unregular pancreatectomy (enucleation, middle pancreatectomy, etc.) was performed; (III) pancreatic or extra-pancreas lesions might influence normal glucose metabolism like insulinoma, glucagonoma, ectopic ACTH syndrome, etc. Patients were separated into four different groups (control group, PD, DP, and TP) by different surgical procedures received. Those who received palliative surgeries without pancreatectomy were categorized to the control group.

\section{CGM system and glucose monitoring}

The FreeStyle Libre (FL; Abbott Diabetes Care, Alameda, CA, USA) flash glucose monitoring system was used in our study for CGM. This device used a small disposable sensor, which should be implanted subcutaneously to measure interstitial glucose values for a maximum of 14 days. After being implanted and fully set, the CGM device will continuously monitor interstitial glucose levels and record the glucose value down for an interval of every 15 minutes.

In this study, the sensor was implanted on the lateral side of the right upper arm for all participants. To avoid potential disturbance brought by electrothermal surgical devices (16), sensors were placed and activated in the postanesthesia care unit (PACU) right after the surgery. After the device had been set for over 14 days or the patient was discharged, the sensor was removed from the patient, and all data were gathered. During the whole process of the study, clinical decisions were made independent of the glucose value recorded by the CGM system.

\section{Perioperative management}

As all patients included were non-diabetic preoperatively, no extra intravenous or subcutaneous insulin was prescribed preoperatively. During the postoperative period, for patients who underwent PD, DP, and TP, 1 unit of insulin was applied with every 3 grams of carbohydrates if there were carbohydrates in the intravenous fluids. For patients in the control group, no intravenous insulin was applied with carbohydrates in fluids. Besides insulin prescribed with fluids, for patients in the control group, PD, and DP, subcutaneous insulin was prescribed on demand to keep the patient reach a target glucose level of 3.9-10 mmol/L. In the TP group, an intravenous insulin pump was used to control glucose until the patient resumed oral intake. After a TP patient resumed oral intake, an insulin replacement therapy strategy combining subcutaneous rapid-acting and long-acting insulin was prescribed. The strategy was adjusted daily according to the glucose status on the previous day. In this study, the daily extra insulin dose during the CGM monitoring period was calculated as the total amount of insulin prescribed except for insulin applied with carbohydrates in intravenous fluids.

Major postoperative complications, including clinically relevant postoperative pancreatic fistula (CR-POPF) (19), delayed gastric empty (DGE) (20), post-pancreatectomy hemorrhage (PPH) (21), SSI (1), and other events met Clavien-Dindo grade 3 or higher (22), were recorded in our study.

\section{Statistical analysis}

The mean and the standard variation (SD) of glucose value was calculated with all glucose values recorded in all patients of each group. To access perioperative glucose variability between different groups, the following several parameters were introduced. The coefficient of variation (CV) of glucose for each group was calculated as the SD divided by the mean of glucose. Mean of daily difference (MODD), reflecting inter-day glucose variation, was defined as the mean of absolute differences between glucose values taken on two sequent days at the same time (23). Continuous overall net glycemic action per $n$ hours (CONGA, $n$ ), a parameter for describing intra-day glucose variation, was defined as the SD of all differences between the current glucose value and the value $n$ hours ago $(23,24)$. For describing the glucose distribution in each group, time above range (TAR) $(>10 \mathrm{mmol} / \mathrm{L})$, time in range $(3.9-10 \mathrm{mmol} / \mathrm{L})$, and time below range (TBR) $(<3.9 \mathrm{mmol} / \mathrm{L})$ was evaluated according to the published international consensus (13).

All statistical analyses were performed with $\mathrm{R}$ (version 4.0.2), and graphic presentations were made in GraphPad Prism 8 (GraphPad Software, San Diego, California, USA). We used R package CGManalyzer to calculate MODD and CONGA for each individual (25), and R package cvequality (version 0.1.3; Marwick and Krishnamoorthy 2019) to calculate CV and test for significant differences between groups. For continuous data, if a variable followed 
Table 1 Preoperative baseline characteristics between different groups

\begin{tabular}{|c|c|c|c|c|c|c|}
\hline & All cases & CTRL & PD & DP & TP & $P$ value \\
\hline Age (years) & $57.11 \pm 13.35$ & $57.0 \pm 17.1$ & $61.9 \pm 11.5$ & $50.6 \pm 15.5$ & $54.5 \pm 12.0$ & 0.554 \\
\hline Height $(\mathrm{cm})$ & $164.11 \pm 5.44$ & $164.00 \pm 5.29$ & $165.25 \pm 6.54$ & $161.60 \pm 4.10$ & $166.00 \pm 5.66$ & 0.686 \\
\hline Weight (kg) & $57.25 \pm 8.41$ & $55.17 \pm 4.75$ & $54.63 \pm 7.31$ & $58.40 \pm 9.36$ & $68.00 \pm 11.31$ & 0.236 \\
\hline FBG (mmol/L) & $5.22 \pm 0.53$ & $4.90 \pm 0.87$ & $5.21 \pm 0.35$ & $5.14 \pm 0.47$ & $5.50 \pm 0.99$ & 0.611 \\
\hline HbA1c (\%) & $5.38 \pm 0.63$ & $5.07 \pm 0.61$ & $5.48 \pm 0.77$ & $5.48 \pm 0.58$ & $5.20 \pm 0.28$ & 0.868 \\
\hline C-peptide $(\mu \mathrm{g} / \mathrm{L})$ & $2.01 \pm 1.22$ & $3.58 \pm 2.35$ & $1.83 \pm 0.58$ & $1.51 \pm 0.79$ & $1.62 \pm 0.28$ & 0.086 \\
\hline HOMA-IR & $2.37 \pm 2.09$ & $4.14 \pm 3.81$ & $2.39 \pm 2.04$ & $1.36 \pm 0.39$ & $2.14 \pm 0.79$ & 0.365 \\
\hline
\end{tabular}

Data are presented as mean \pm standard deviation. BMI, body mass index; FBG, fasting blood glucose; HOMR-IR, homeostatic model assessment for insulin resistance; IU, international units; POD, postoperative days of stary before discharge; CTRL, control group; PD, pancreaticoduodenectomy; DP, distal pancreatectomy; TP, total pancreatectomy.

the Gaussian distribution, one-way ANOVA was used to compare group differences; otherwise, the Kruskal-Wallis test was applied. For categorized data, chi-square test was applied. Continuous data were presented as mean $\pm \mathrm{SD}$ if applicable. $\mathrm{P}$ values $<0.05$ were accepted as statistically significant.

\section{Results}

\section{Baseline characteristics of all participants}

A total of 20 participants were initially recruited, 18 were finally included in further evaluation. Two patients dropped out of the study, one was excluded due to a technical failure of the sensor, and the other withdrew the informed consent before discharge. A flow diagram of participants recruited was provided in Figure S1.

Among 18 patients finally included, 2 received TP, 8 received PD (the Whipple procedure), 5 received DP, and 3 received palliative surgery without pancreatectomy ( 2 palliative bypass surgery and 1 "open-close" laparotomy). The pathological diagnosis of these patients included 14 pancreatic ductal adenocarcinoma (PDAC), 1 intraductal papillary mucinous neoplasm (IPMN), 1 serous cystic neoplasm (SCN), 1 periampullary carcinoma, and 1 multiple myeloma (Table S1). The average length of
CGM monitoring for all participants was $10.00 \pm 3.40$ days.

The baseline characteristics of all participants for each group were listed in Table 1. For patients in four different groups, they had no statistically significant difference in gender distribution $(\mathrm{P}=0.727)$. They had similar results in average age, height, weight, body mass index (BMI), fasting blood glucose (FBG), HbA1c, C-peptide, and homeostatic model assessment for insulin resistance (HOMA-IR) index (all $\mathrm{P}$ value $>0.05$ ).

The average postoperative days of stay before discharge was $11.94 \pm 6.70$ days, and no significant difference was noticed between all four groups $(\mathrm{P}=0.273)$. For postoperative daily extra insulin dose during the monitoring period, the TP group had the highest dose of $28.60 \pm 12.03$ units, the control group had the lowest dose of $0.10 \pm 0.18$ units $(\mathrm{P}<0.001)$. The PD and the DP group had a similar dose of extra insulin, and further pairwise comparison found no significant difference between these two groups $(\mathrm{P}>0.05)$.

The overall incidence of postoperative complications was low. The control group and the TP group had no complications. CR-POPF occurred only on 1 patient in the DP group, and biochemical leak happened on 6 patients (2 in PD, 4 in DP). Grade C DGE happened on 2 patients in the PD group. Superficial SSI occurred on 1 patient in the PD group. 
Table 2 Postoperative glycemic variability between different groups

\begin{tabular}{lcccc}
\hline & CTRL & PD & DP & TP \\
\hline Mean glucose value & $4.61 \pm 1.61$ & $6.20 \pm 2.26$ & $6.16 \pm 2.07$ & $8.24 \pm 3.76$ \\
CV of mean glucose & 0.349 & 0.365 & 0.336 & 0.457 \\
CONGA, 2 & $1.492 \pm 0.569$ & $1.786 \pm 0.420$ & $1.764 \pm 0.318$ & $2.164 \pm 0.536$ \\
MODD & $0.437 \pm 0.272$ & $0.401 \pm 0.082$ & $0.409 \pm 0.042$ & $0.506 \pm 0.147$ \\
\hline
\end{tabular}

Data are presented as mean \pm standard deviation if applicable. CV, coefficient of variation; CONGA, continuous overall net glycemic action; MODD, mean of daily difference; CTRL, control group; PD, pancreaticoduodenectomy; DP, distal pancreatectomy; TP, total pancreatectomy.

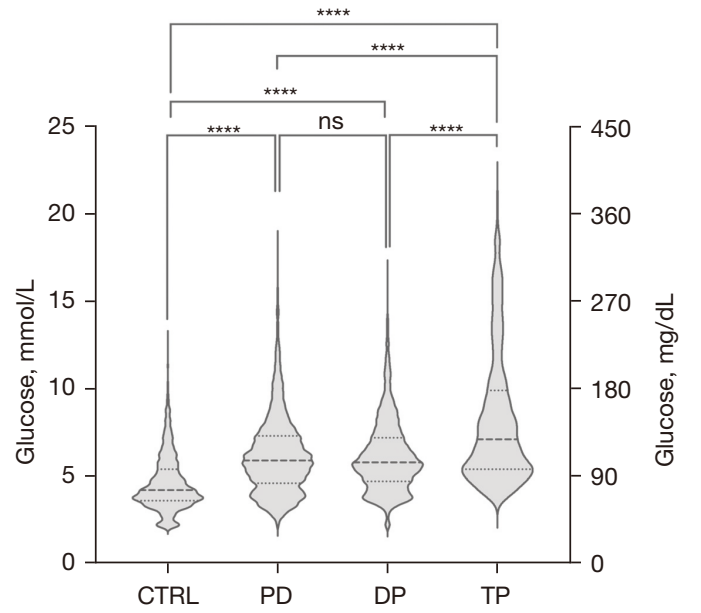

Figure 1 The perioperative mean glucose level and deviation for different groups. ${ }^{* * *}, \mathrm{P}<0.001$. CTRL, control group; $\mathrm{PD}$, pancreaticoduodenectomy; DP, distal pancreatectomy; TP, total pancreatectomy; ns, not significant.

\section{Perioperative glucose level and glycemic variability}

The data about perioperative mean glucose value (MGV) and glycemic variability for all groups were presented in Table 2. As expected, the MGV for the control group was $4.61 \pm 1.61 \mathrm{mmol} / \mathrm{L}$, which was the lowest, while the TP group had the highest MGV of $8.24 \pm 3.76 \mathrm{mmol} / \mathrm{L}$. The PD group and the DP group had similar MGVs $(6.20 \pm 2.26 \mathrm{mmol} / \mathrm{L}$ for $\mathrm{PD}, 6.16 \pm 2.07 \mathrm{mmol} / \mathrm{L}$ for $\mathrm{DP})$ with no significant difference $(\mathrm{P}=0.098)$. The further pairwise comparison suggested, except for the difference between $\mathrm{PD}$ and $\mathrm{DP}$, other differences between groups were all significant $(\mathrm{P}<0.001)$ (Figure 1, Table $\mathrm{S} 2)$.

Furthermore, the TP group had the highest CV of 0.457 , indicating patients who underwent TP were characterized by greater postoperative glucose fluctuation than others.
Other three groups had similar $\mathrm{CV}$ values (Control: 0.349; PD: 0.365; DP: 0.336). Nevertheless, statistical analysis suggested the pairwise differences of $\mathrm{CV}$ between groups were all significant (Table S2). Figure 2 presents the difference in glucose fluctuation per 24-hour period between the control group and other groups.

For net glycemic variation accessed by CONGA, 2, the TP group exhibited the highest value of $2.164 \pm 0.536$, and the control group had the lowest value of $1.492 \pm 0.569$. Meanwhile, PD and DP had similar values between TP and the control group (PD: $1.786 \pm 0.420$; DP: $1.764 \pm 0.318$ ). However, no statistically significant differences were found between groups. For MODD, the TP group had the highest value $(0.506 \pm 0.147)$, followed by the control group, DP, and PD. But there were also no statistically significant differences between groups.

\section{The distribution of postoperative glucose value}

The glucose distribution of each group was listed in Table 3 and graphically presented in Figure 3. In all four groups, the TP group spent the most time above the target range $(24.29 \%)$ and the least time below the range (1.28\%). In contrast, the control group bare have glucose value above range $(0.79 \%)$ and spent the most time below the range (37.01\%). Pairwise comparisons between groups about time above/below range were presented in Table S3, all $\mathrm{P}$ values $<0.05$.

\section{Other potential factors that might affect glucose status}

Besides the difference between different surgical procedures, we also tried to explore the potential effect of 2 other factors that might have an impact on postoperative glucose status.

Firstly, postoperative complications were taken into 
A

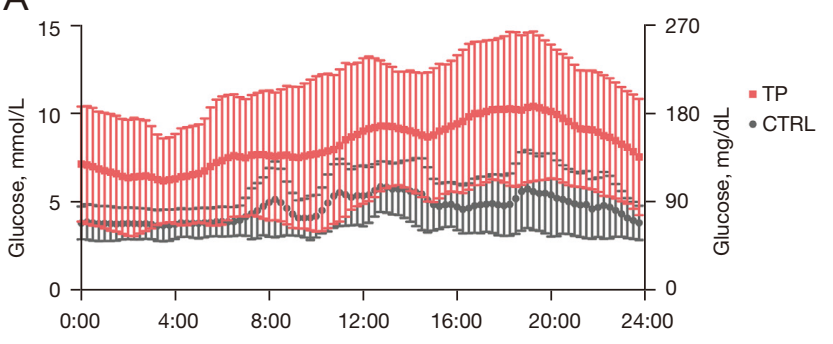

C

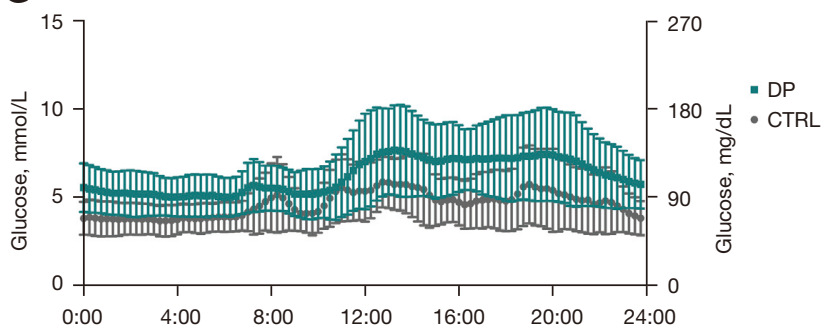

B

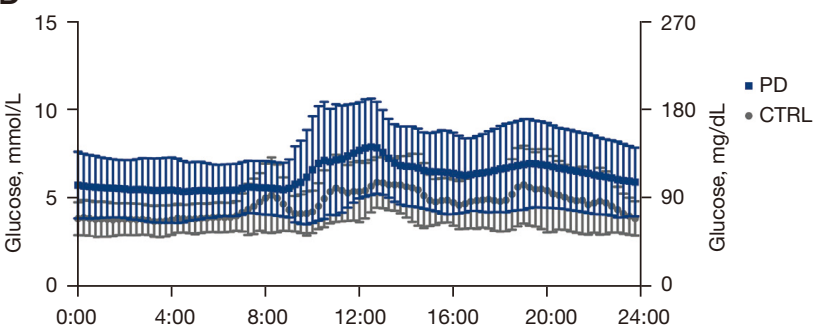

Figure 2 Comparisons of glucose fluctuation per 24-hour period between the control and other groups. (A) The control group (CTRL, grey) vs. total pancreatectomy (TP, red). (B) The control group (CTRL, grey) vs. pancreaticoduodenectomy (PD, blue); (C) The control group (CTRL, grey) vs. distal pancreatectomy (DP, green). CTRL, control group; PD, pancreaticoduodenectomy; DP, distal pancreatectomy; TP, total pancreatectomy.

Table 3 The distribution of postoperative glucose value between different groups

\begin{tabular}{|c|c|c|c|c|c|}
\hline & CTRL & PD & DP & TP & $P$ value \\
\hline Time within range (3.9-10 mmol/L) (\%) & 62.20 & 79.95 & 83.62 & 74.43 & \\
\hline Time above range (>10 mmol/L) (\%) & 0.79 & 6.65 & 5.53 & 24.29 & \\
\hline
\end{tabular}

CTRL, control group; PD, pancreaticoduodenectomy; DP, distal pancreatectomy; TP, total pancreatectomy.

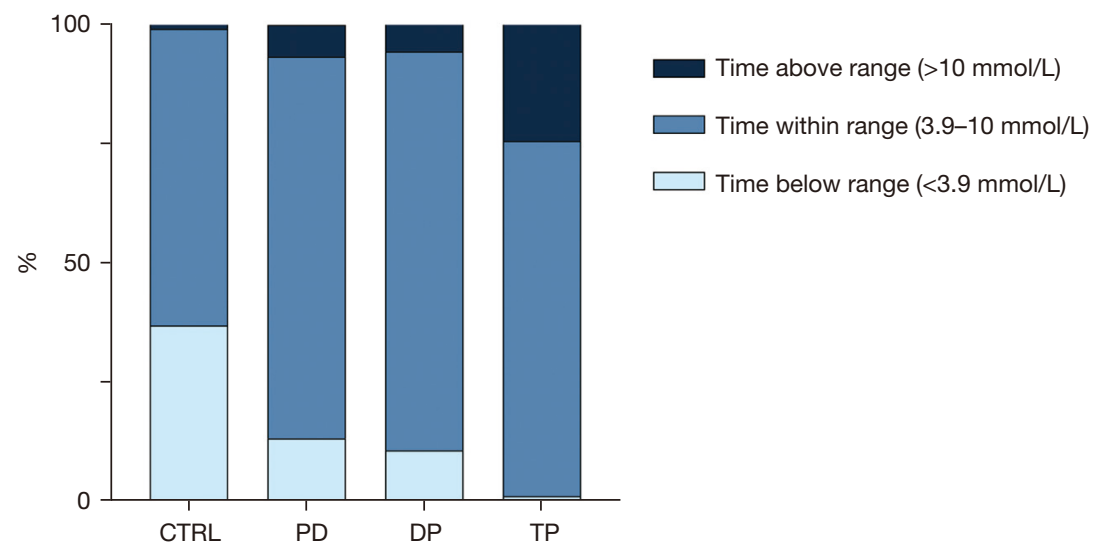

Figure 3 The glucose distribution of different groups. CTRL, control group; PD, pancreaticoduodenectomy; DP, distal pancreatectomy; $\mathrm{TP}$, total pancreatectomy. 
consideration. On this topic, we only took data from PD and DP groups to avoid potential bias caused by different surgical procedures. Results suggested that patients without any grades of POPF seemed to have a higher mean glucose level than those with POPF $(6.68 \pm 2.24$ vs. $5.77 \pm 1.98$, $\mathrm{P}<0.001)$, but they had no significant difference in glycemic variability (Table S4). A similar result was also found between patients with and without major complications $(6.17 \pm 1.96$ vs. $6.03 \pm 2.50, \mathrm{P}<0.001)$ (Table S5).

Secondly, the difference between the first 5 days and 5 days after surgery was investigated (Table S6). PD and DP group had a higher mean glucose level in the first 5 days, while the TP group showed a higher mean glucose level after 5 days (all $\mathrm{P}$ values $<0.001$ ).

\section{Discussion}

As pancreatic surgeries have been performed more than before, NODM or the glycemic change after pancreatectomy is now attracting greater attention from both surgeons and physicians $(7,8,10,26,27)$. Nevertheless, neither the pathophysiology nor the pathogenesis behind it has been fully understood, and only a few studies have demonstrated the detailed glycemic variability after pancreatectomy. CGM device provides us a utility tool for glucose monitoring $(13,28)$. Recently, there were two studies using CGM to investigate patients' glycemic status and variation long after the original pancreatic surgery $(7,8)$. However, perioperative glycemic variability seems to have never been described for pancreatic surgeries. Our work might be the first study using CGM to characterize the perioperative glucose level and glycemic variability of different types of pancreatic surgeries.

The results of our study revealed that patients after total pancreatectomy had the highest mean glucose level, the greatest glycemic variability, and the longest time above the target range in all four groups. Those who underwent PD or DP had similar mean glucose levels, which were higher than the control group and lower than the TP group. Furthermore, these two groups seemed to have a nearnormal glucose variability resembling the control group.

All patients who received any type of pancreatectomy had a higher mean glucose level than control. This could be mainly explained by the loss of beta cells, leading to the definite insufficiency of intrinsic insulin. TP led to the loss of all intrinsic insulin, mimicking patients with type 1 diabetes mellitus (8), thus presented the highest mean glucose level. In the meanwhile, TP also depleted all alpha cells and completely broke down the existing glucose hemostatic system, making patients insufficient for both glucose-raising and glucose-lowering hormones. Besides, patients who received TP were related to increased peripheral insulin sensitivity and decreased hepatic reaction to insulin $(29,30)$. These made the TP group also presents the most significant glycemic variability assessed by $\mathrm{CV}$, CONGA, and MODD, which was in accordance with the clinical impression of "brittle" diabetes when talking about NODM after pancreatectomy (30).

When it comes to PD and DP, they had a higher mean glucose level than control but lower than TP. The finding was consistent with the fact that PD and DP were only related to partial depletion of pancreas parenchyma and beta cells. At the same time, they also seemed to have a near-normal glycemic variability resembling the control group, reflecting these two groups could still somehow maintain glucose hemostasis to some certain extent under the circumstance of insulin deficiency. This might partially be attributed to the function of other endocrine cells in the Langerhans islet (31-33).

Interestingly, existent evidence suggested that the tail of the pancreas usually had a higher density of beta cells than the head of the pancreas (34). Therefore, patients who received DP were supposed to suffer more from insulin insufficiency than PD. However, our study found no difference between these two groups. This might remind us there might be other factors besides beta-cell loss that could affect postoperative glucose metabolism. In fact, the exact volume of resected pancreas parenchyma (10), gastrointestinal anastomosis $(35,36)$, intestinal microbes $(7,37,38)$ and so on were all possible factors. These factors might also contribute to maintaining a near-normal glucose variability after PD and DP. Further studies could make a more elaborate design to investigate the effect of each possible factor on glucose metabolism after different types of pancreatic surgeries.

In this study, we also found patients in the control group experienced the largest percentage of TBR, leading to an illusion that the control group might have a higher chance of suffering hypoglycemic attacks. However, we consider this result was not clinically significant because the normal range of glucose level could extend down to $3 \mathrm{mmol} / \mathrm{L}$ (39) and interstitial glucose detected by CGM was usually lower than capillary or venous glucose $(16,40)$. Therefore, a glucose level below the target range ( $4 \mathrm{mmol} / \mathrm{L}$ ) but above $3 \mathrm{mmol} / \mathrm{L}$ could be regarded as normal values for patients in our control group. 
We also tried to investigate the effect of postoperative complications on glycemic status. Our results suggest that patients with complications were related to a lower average glucose level, which seemed just opposite to the hypothesis that hyperglycemia could lead to a poor surgical outcome (1-3). Our team considered that our postoperative management might cause the difference. For instance, when a patient had already or was about to develop comorbidities, we always started to give the patient more intensive glucose management than the previous period. Meanwhile, after a complication developed, discharging was delayed. These factors made patients with complications receive intensive glucose management for longer, finally leading to a lower average glucose level.

In addition, we also found PD and DP had higher mean glucose in the first 5 days while TP showed the opposite. We assumed that the PD and DP were caused by postoperative stress and intravenous fluids in the first 3 to 5 days after surgery. When it comes to TP, although postoperative stress and fluids do play a role, the higher mean glucose after 5 days might reflect that oral diets could cause a more significant effect and exceed what was caused by fluids or stress. As these two results were extracted from limited data, they should be carefully interpreted to other fields and re-confirmed by future studies.

Through the current study, for the first time, we acquired a preliminary acknowledgment for the general pattern of perioperative glycemic status after three major types of pancreatic surgeries. The result might help surgeons to optimize the strategy of perioperative glucose management. The diurnal glucose fluctuation provided by CGM like Figure 2 could help us find some interesting and easily overlooked factors, which would never be detected when using traditional point of care tests. According to the findings, different strategies of insulin replacement therapy and glucose monitoring could be tailored for different types of surgery. For example, patients after TP might need a higher total dose of daily insulin to reach optimal glucose control as they presented a higher mean glucose level. Moreover, to avoid acute complications related to hypoor hyperglycemia, they might also need more intensive glucose monitoring and avoid bolus insulin administration due to the more significant glycemic variability. Besides, CGM devices might be more suitable for these patients. It can provide the patient with more meticulous care of glucose while bringing no extra painful experiences, and it can also reduce the workload and the burden for nurses (16). Meanwhile, patients who received PD or DP might share a similar strategy during the perioperative period. Additional insulin replacement might help them to maintain the glucose level in the optimal range, and a regular point-ofcare approach would be enough for glucose monitoring.

Furthermore, revealing perioperative glycemic status for different surgeries might help explore the pathogenesis and pathophysiology of NODM after pancreatectomy. Comparing results between different surgical groups might help us to recognize different triggers of NODM related to specific surgical procedures. Comparing perioperative glycemic status with results long after initial surgery might discover compensation mechanisms for regaining glucose hemostasis. For instance, in a recent study, researchers found that patients who underwent DP were more vulnerable to diabetes than PD (7), whereas our study found almost no difference between these two groups. This indicating there might be one or more "late triggers" developed during the long postoperative period, which might be discovered in future studies.

As it was only a pilot observational study, our study still had several limitations. First, the study had a relatively small sample size, and subjects were not evenly distributed in each group. Some results might not show a statistically significant difference due to this limitation. For example, our study found a trend of CONGA was higher in PD/DP than the control and was highest in TP, which was in line with clinical experience, but statistical analysis showed no significant difference. Another limitation was our control group was not that a "perfect" control group. As "no pancreatectomy" was the only inclusion criteria, patients in this group received different palliative surgeries, thus making the control group somehow lacked internal consistency. As no pancreatectomy was performed, the control group usually resumed oral intake earlier than others and had no limitations on diets. This made them have less external consistency and present higher inter-day glucose variation than others due to the influence of diets. Therefore, in our results, the control group had a higher MODD than PD and DP. Besides the patients in the control group were at a late stage of pancreatic cancer and were unresectable, which was distinct from the other three groups, and this might give additional bias to the results. To solve these issues, our future studies will focus on only one or two specific types of pancreatic surgery, include a greater number of participants, and choose a more suitable control group for it. Finally, it was a pity that we failed to give most of these patients a proper longterm follow-up, so the long-term glycemic status between different surgical procedures was unknown. Now we are 
conducting a new study on this topic.

\section{Conclusions}

Our study first attempted to describe the perioperative glucose level and glycemic variability after three different types of pancreatic surgeries with the CGM system. TP group had the highest mean glucose level and the greatest glycemic variability. PD and DP had similar results: a higher mean glucose level than control but lower than TP. For glycemic variability, PD and DP seemed to have a nearnormal result resembling the control group.

\section{Acknowledgments}

The authors would like to thank all participants.

Funding: This work was supported by grants from the National Natural Science Foundation of China (No. 81772566 to J.L.); and the Innovation Program in Artificial Intelligence from Shanghai Municipal Committee of Science \& Technology (No. 19511121200 to J.L.).

\section{Footnote}

Reporting Checklist: The authors have completed the STROBE reporting checklist. Available at https://dx.doi. org/10.21037/gs-21-495

Data Sharing Statement: Available at https://dx.doi. org/10.21037/gs-21-495

Peer Review File: Available at https://dx.doi.org/10.21037/ gs-21-495

Conflicts of Interest: All authors have completed the ICMJE uniform disclosure form (available at https://dx.doi. org/10.21037/gs-21-495). The authors have no conflicts of interest to declare except the funding.

Ethical Statement: The authors are accountable for all aspects of the work in ensuring that questions related to the accuracy or integrity of any part of the work are appropriately investigated and resolved. The study was conducted in accordance with the Declaration of Helsinki (as revised in 2013). The study was approved by the Clinical Ethics Committee of Huashan Hospital and registered at chictr.org.cn (ChiCTR2000030177). Informed consent was taken from all individual participants.
Open Access Statement: This is an Open Access article distributed in accordance with the Creative Commons Attribution-NonCommercial-NoDerivs 4.0 International License (CC BY-NC-ND 4.0), which permits the noncommercial replication and distribution of the article with the strict proviso that no changes or edits are made and the original work is properly cited (including links to both the formal publication through the relevant DOI and the license). See: https://creativecommons.org/licenses/by-nc-nd/4.0/.

\section{References}

1. Berríos-Torres SI, Umscheid CA, Bratzler DW, et al. Centers for Disease Control and Prevention Guideline for the Prevention of Surgical Site Infection, 2017. JAMA Surg 2017;152:784-91.

2. Sebranek JJ, Lugli AK, Coursin DB. Glycaemic control in the perioperative period. Br J Anaesth 2013;111 Suppl 1:118-34.

3. Kotagal M, Symons RG, Hirsch IB, et al. Perioperative hyperglycemia and risk of adverse events among patients with and without diabetes. Ann Surg 2015;261:97-103.

4. Okabayashi T, Shima $Y$, Sumiyoshi T, et al. Intensive versus intermediate glucose control in surgical intensive care unit patients. Diabetes Care 2014;37:1516-24.

5. Lee BW, Kang HW, Heo JS, et al. Insulin secretory defect plays a major role in the development of diabetes in patients with distal pancreatectomy. Metabolism 2006;55:135-41.

6. American Diabetes Association. Diagnosis and classification of diabetes mellitus. Diabetes Care 2012;35 Suppl 1:S64-71.

7. Fukuda T, Bouchi R, Takeuchi T, et al. Importance of Intestinal Environment and Cellular Plasticity of Islets in the Development of Postpancreatectomy Diabetes. Diabetes Care 2021;44:1002-11.

8. Juel CTB, Dejgaard TF, Hansen CP, et al. Glycemic Control and Variability of Diabetes Secondary to Total Pancreatectomy Assessed by Continuous Glucose Monitoring. J Clin Endocrinol Metab 2021;106:168-73.

9. Maker AV, Sheikh R, Bhagia V, et al. Perioperative management of endocrine insufficiency after total pancreatectomy for neoplasia. Langenbecks Arch Surg 2017;402:873-83.

10. Kwon JH, Kim SC, Shim IK, et al. Factors Affecting the Development of Diabetes Mellitus After Pancreatic Resection. Pancreas 2015;44:1296-303.

11. De Bruijn KM, van Eijck CH. New-onset diabetes after 
distal pancreatectomy: a systematic review. Ann Surg 2015;261:854-61.

12. Ji L, Guo X, Guo L, et al. A Multicenter Evaluation of the Performance and Usability of a Novel Glucose Monitoring System in Chinese Adults With Diabetes. J Diabetes Sci Technol 2017;11:290-5.

13. Danne T, Nimri R, Battelino T, et al. International Consensus on Use of Continuous Glucose Monitoring. Diabetes Care 2017;40:1631-40.

14. Kestilä KK, Ekblad UU, Rönnemaa T. Continuous glucose monitoring versus self-monitoring of blood glucose in the treatment of gestational diabetes mellitus. Diabetes Res Clin Pract 2007;77:174-9.

15. Song IK, Lee JH, Kang JE, et al. Continuous glucose monitoring system in the operating room and intensive care unit: any difference according to measurement sites? J Clin Monit Comput 2017;31:187-94.

16. Perez-Guzman MC, Duggan E, Gibanica S, et al. Continuous Glucose Monitoring in the Operating Room and Cardiac Intensive Care Unit. Diabetes Care 2021;44:e50-2.

17. Agarwal S, Mathew J, Davis GM, et al. Continuous Glucose Monitoring in the Intensive Care Unit During the COVID-19 Pandemic. Diabetes Care 2021;44:847-9.

18. Sugiyama $Y$, Wakabayashi R, Urasawa $M$, et al. Perioperative Characteristics of the Accuracy of Subcutaneous Continuous Glucose Monitoring: Pilot Study in Neurosurgery and Cardiac Surgery. Diabetes Technol Ther 2018;20:654-61.

19. Bassi C, Marchegiani G, Dervenis C, et al. The 2016 update of the International Study Group (ISGPS) definition and grading of postoperative pancreatic fistula: 11 Years After. Surgery 2017;161:584-91.

20. Wente MN, Bassi C, Dervenis C, et al. Delayed gastric emptying (DGE) after pancreatic surgery: a suggested definition by the International Study Group of Pancreatic Surgery (ISGPS). Surgery 2007;142:761-8.

21. Wente MN, Veit JA, Bassi C, et al. Postpancreatectomy hemorrhage (PPH): an International Study Group of Pancreatic Surgery (ISGPS) definition. Surgery 2007;142:20-5.

22. Dindo D, Demartines N, Clavien PA. Classification of surgical complications: a new proposal with evaluation in a cohort of 6336 patients and results of a survey. Ann Surg 2004;240:205-13.

23. McDonnell CM, Donath SM, Vidmar SI, et al. A novel approach to continuous glucose analysis utilizing glycemic variation. Diabetes Technol Ther 2005;7:253-63.
24. Service FJ. Glucose variability. Diabetes 2013;62:1398-404.

25. Zhang XD, Zhang Z, Wang D. CGManalyzer: an R package for analyzing continuous glucose monitoring studies. Bioinformatics 2018;34:1609-11.

26. Ishibashi C, Kozawa J, Fujita Y, et al. Glucose Intolerance After Pancreatectomy Was Associated With Preoperative Hemoglobin A1c, Insulin Resistance, and Histological Pancreatic Fatty Infiltration. Pancreas 2018;47:e48-50.

27. Beger HG, Poch B, Mayer B, et al. New Onset of Diabetes and Pancreatic Exocrine Insufficiency After Pancreaticoduodenectomy for Benign and Malignant Tumors: A Systematic Review and Meta-analysis of Longterm Results. Ann Surg 2018;267:259-70.

28. Perez-Guzman MC, Shang T, Zhang JY, et al. Continuous Glucose Monitoring in the Hospital. Endocrinol Metab (Seoul) 2021;36:240-55.

29. Pliam MB, ReMine WH. Further evaluation of total pancreatectomy. Arch Surg 1975;110:506-12.

30. Maeda H, Hanazaki K. Pancreatogenic diabetes after pancreatic resection. Pancreatology 2011;11:268-76.

31. Schrader H, Menge BA, Breuer TG, et al. Impaired glucose-induced glucagon suppression after partial pancreatectomy. J Clin Endocrinol Metab 2009;94:2857-63.

32. Bajorunas DR, Fortner JG, Jaspan J, et al. Total pancreatectomy increases the metabolic response to glucagon in humans. J Clin Endocrinol Metab 1986;63:439-46.

33. Andersen DK. Mechanisms and emerging treatments of the metabolic complications of chronic pancreatitis. Pancreas 2007;35:1-15.

34. Wittingen J, Frey CF. Islet concentration in the head, body, tail and uncinate process of the pancreas. Ann Surg 1974;179:412-4.

35. Mori Y, Ohtsuka T, Tsutsumi K, et al. Different incretin responses after pancreatoduodenectomy and distal pancreatectomy. Pancreas 2012;41:455-60.

36. Jirapinyo P, Haas AV, Thompson CC. Effect of the Duodenal-Jejunal Bypass Liner on Glycemic Control in Patients With Type 2 Diabetes With Obesity: A Metaanalysis With Secondary Analysis on Weight Loss and Hormonal Changes. Diabetes Care 2018;41:1106-15.

37. Barlow GM, Yu A, Mathur R. Role of the Gut Microbiome in Obesity and Diabetes Mellitus. Nutr Clin Pract 2015;30:787-97.

38. Knip M, Siljander H. The role of the intestinal microbiota in type 1 diabetes mellitus. Nat Rev Endocrinol 2016;12:154-67. 
39. International Hypoglycaemia Study Group. Glucose Concentrations of Less Than $3.0 \mathrm{mmol} / \mathrm{L}$ (54 mg/dL) Should Be Reported in Clinical Trials: A Joint Position Statement of the American Diabetes Association and the European Association for the Study of Diabetes. Diabetes
Care 2017;40:155-7.

40. Schierenbeck F, Franco-Cereceda A, Liska J. Accuracy of 2 Different Continuous Glucose Monitoring Systems in Patients Undergoing Cardiac Surgery. J Diabetes Sci Technol 2017;11:108-16.

Cite this article as: Mao Y, Zhao X, Zhou L, Lu B, Jin C, Fu D, Yao L, Li J. Evaluating perioperative glycemic status after different types of pancreatic surgeries via continuous glucose monitoring system: a pilot study. Gland Surg 2021;10(10):29452955. doi: $10.21037 /$ gs-21-495 


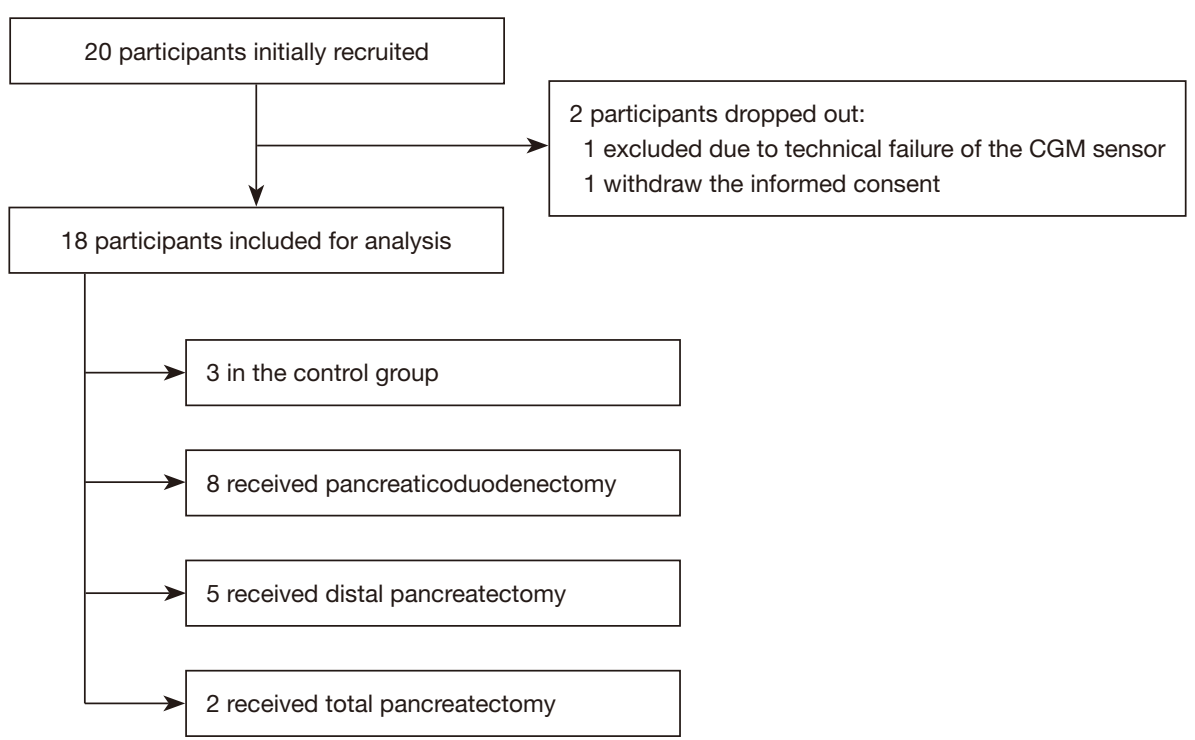

Figure S1 The flow diagram for participants recruited.

Table S1 The pathological diagnosis and surgery for each patient

\begin{tabular}{|c|c|c|c|c|}
\hline \multicolumn{2}{|c|}{ Patient Diagnosis } & $\begin{array}{l}\text { Surgery } \\
\text { DP }\end{array}$ & $\begin{array}{l}\text { Resection line } \\
\text { Above PV }\end{array}$ & $\frac{\text { Average daily extra insulin (IU) }}{3.80}$ \\
\hline 2 & PDAC & PD & Above PV & 2.00 \\
\hline 3 & IPMN & PD & Above PV & 0 \\
\hline 4 & PDAC & TP & $\mathrm{N} / \mathrm{A}$ & 20.09 \\
\hline 6 & PDAC & $\mathrm{DP}$ & Above PV & 2.44 \\
\hline 7 & PDAC & $\mathrm{DP}$ & Above PV & 0.57 \\
\hline 8 & multiple myeloma & PD & Above PV & 0.57 \\
\hline 9 & PDAC & $\mathrm{DP}$ & Above PV & 0 \\
\hline 12 & PDAC & TP & $\mathrm{N} / \mathrm{A}$ & 37.10 \\
\hline 13 & PDAC & Palliative gastrojejunostomy and cholangiojejunosotmy & $\mathrm{N} / \mathrm{A}$ & 0 \\
\hline 14 & PDAC & PD & Above PV & 0.86 \\
\hline 15 & PDAC & Palliative gastrojejunostomy & N/A & 0 \\
\hline 16 & PDAC & PD & Above PV & 2.17 \\
\hline 17 & PDAC & $\begin{array}{l}\text { "Open-close" laparotomy with biopsy for metastatic } \\
\text { lesions on peritoneum }\end{array}$ & $\mathrm{N} / \mathrm{A}$ & 0.31 \\
\hline 18 & PDAC & PD & Above PV & 0.86 \\
\hline
\end{tabular}

PDAC, pancreatic ductal adenocarcinoma; IPMN, intraductal papillary mucinous neoplasm; SCN, serous cystic neoplasm; PD, pancreaticoduodenectomy; DP, distal pancreatectomy; TP, total pancreatectomy; PV, portal vein; N/A, not applicable; IU, international units. 
Table S2 Results of pairwise comparison of postoperative glycemic variability different groups

\begin{tabular}{lcc}
\hline & Mean glucose value & CV of mean glucose \\
\hline CTRL vs. PD & $<0.001$ & 0.009 \\
CTRL vs. DP & $<0.001$ & 0.030 \\
CTRL vs. TP & $<0.001$ & $<0.001$ \\
TP vs. PD & $<0.001$ & $<0.001$ \\
TP vs. DP & $<0.001$ & $<0.001$ \\
PD vs. DP & 0.980 & $<0.001$
\end{tabular}

CTRL, control group; PD, pancreaticoduodenectomy; DP, distal pancreatectomy; TP, total pancreatectomy.
Table S3 Results of pairwise comparison of the distribution of postoperative glucose value between different groups

\begin{tabular}{lcc} 
& Time above range & Time below range \\
\hline CTRL vs. PD & $<0.001$ & $<0.001$ \\
CTRL vs. DP & $<0.001$ & $<0.001$ \\
CTRL vs. TP & $<0.001$ & $<0.001$ \\
TP vs. PD & $<0.001$ & $<0.001$ \\
TP vs. DP & $<0.001$ & $<0.001$ \\
PD vs. DP & 0.012 & $<0.001$ \\
\hline
\end{tabular}

CTRL, control group; PD, pancreaticoduodenectomy; DP, distal pancreatectomy; TP, total pancreatectomy.

Table S4 The potential effect of post-operative complication (POPF) on patients' glycemic status in PD and DP

\begin{tabular}{lccc}
\hline & With POPF $(\mathrm{n}=7)$ & Without POPF $(\mathrm{n}=6)$ & $\mathrm{P}$ value \\
\hline Mean glucose value & $5.77 \pm 1.98$ & $6.68 \pm 2.24$ & 0.3354 \\
CV of mean glucose & 0.3427 & $1.88 \pm 0.40$ & 0.138 \\
CONGA, 2 & $1.69 \pm 0.35$ & 0.705 \\
MODD & $0.38 \pm 0.05$ & $0.42 \pm 0.08$ & 0.353 \\
\hline
\end{tabular}

POPF included all levels of POPF: biochemic leak, grade B, and grade C. POPF, post-operative pancreatic fistula; PD, pancreaticoduodenectomy; DP, distal pancreatectomy; CV, coefficient of variation; CONGA, continuous overall net glycemic action; MODD, mean of daily difference.

Table S5 The potential effect of post-operative complication (major complications) on patients' glycemic status in PD and DP

\begin{tabular}{lccc}
\hline & With complications $(\mathrm{n}=4)$ & Without complications $(\mathrm{n}=9)$ & $\mathrm{P}$ value \\
\hline Mean glucose value & $6.03 \pm 2.50$ & $6.17 \pm 1.96$ & $<.3175$ \\
CV of mean glucose & 0.4151 & $1.78 \pm 0.31$ & $<0.001$ \\
CONGA, 2 & $1.78 \pm 0.54$ & 0.179 \\
MODD & $0.42 \pm 0.10$ & $0.40 \pm 0.06$ & 0.212 \\
\hline
\end{tabular}

Major complications were defined as clinical relevant post-operative pancreatic fistula (CR-POPF), all grades of delayed gastric empty (DGE), all grades of post-pancreatectomy hemorrhage (PPH), all grades of surgical site infection (SSI), and all other Clavien-Dindo $\geq 3$ events. PD, pancreaticoduodenectomy; DP, distal pancreatectomy; CV, coefficient of variation; CONGA, continuous overall net glycemic action; MODD, mean of daily difference.

Table S6 The difference of mean glucose level (mmol/L) within and after 5 days in each group

\begin{tabular}{lccc}
\hline & First 5 days & After 5 days & P value \\
\hline PD & $6.67 \pm 2.07$ & $5.71 \pm 2.24$ & $<0.001$ \\
DP & $6.28 \pm 2.09$ & $6.02 \pm 1.94$ & $<0.001$ \\
TP & $8.02 \pm 3.21$ & $8.60 \pm 4.13$ & $<0.001$ \\
\hline
\end{tabular}

PD, pancreaticoduodenectomy; DP, distal pancreatectomy; TP, total pancreatectomy. 